\title{
Auditory Training for Spatial Hearing Loss: Two Case Reports of the Older Adults Who Wear Hearing Aids
}

\author{
Joon Shin ${ }^{1}$, Junghwa Bahng ${ }^{1,2}$ \\ ${ }^{1}$ Department of Audiology and Speech Language Pathology, Hallym University of Graduate Studies, Seoul, Korea \\ ${ }^{2}$ HUGS Center for Hearing and Speech Research, Seoul, Korea
}

\author{
Received: April 7, 2021 \\ Revised: April 30, 2021 \\ Accepted: May 17, 2021 \\ Correspondence: \\ Junghwa Bahng, PhD \\ Department of Audiology and \\ Speech Language Pathology, Hallym \\ University of Graduate Studies, 427 \\ Yeoksam-ro, Gangnam-gu, Seoul \\ 06197, Korea \\ Tel: $+82-70-8638-6833$ \\ Fax: +82-2-3453-6618 \\ E-mail: bahng.jh@hallym.ac.kr
}

\begin{abstract}
The elderly with hearing loss have often experienced spatial hearing deficits when only wearing hearing aids. This case study aimed to investigate whether auditory training for spatial hearing loss could improve the spatial hearing ability of the elderly who wear hearing aids. Two older adults (males, aged 75 years and 78 years) who were wearing bilateral hearing aids participated. They had symmetric sensorineural hearing loss. A total of 16 auditory-training sessions (40 minutes, twice per week) for auditory training for spatial hearing ability provided. For outcome measurements, we assessed speech reception thresholds (SRTs) and spatial release from masking (SRM), using two types of noise, steady-state noise and speech-like noise at pre- and post-auditory training. Both subjects increased SRTs after the auditory training, but the SRM of only one participant improved. Also, there were no masking effects for SRM in terms of noise types. Both subjects reported that their trained hearing skills helped in communication with their families and friends. These results suggested that audiologists can consider auditory training for focusing on the spatial hearing loss for the elderly hearing aids users.
\end{abstract}

Key Words: Aged, Hearing aids, Hearing loss, Signal-to-noise ratio, Spatial processing.

\section{INTRODUCTION}

It is well known that spatial hearing plays a critical role in detecting and understanding speech in complex acoustic environments (Koehnke \& Besing, 2001). Spatial hearing is a specific acoustical and perceptual mechanism in the human brain to selectively attend to the wanted speech in the presence of competing talkers or background noise (Neher et al., 2009). Also, its function depends on how the brain integrates two primary binaural cues, namely interaural intensity difference (IID) and interaural time difference (ITD), of signals reaching each ear (Akeroyd \& Whitmer, 2011). For example, if a person has a problem processing these cues due to hearing loss and aging, she or he can have difficulty understanding speech while simultaneously filtering out competing noises arriving from other directions (Glyde et al., 2013).

(c) This is an Open Access article distributed under the terms of the Creative Commons Attribution Non-Commercial License (https://creativecommons.org/ licenses/by-nc/4.0) which permits unrestricted non-commercial use, distribution, and reproduction in any medium, provided the original work is properly cited.
Unfortunately, current hearing aids have a limitation to improve the spatial cues (Ahlstrom et al., 2009), and the spatial hearing ability of their users did not increase even after the speech is amplified (Glyde et al., 2013). In other words, the hearing-impaired listeners have difficulties due to spatial hearing loss/deficit by only wearing hearing aids, consequently that they still have a problem in understanding speech in noise. As one of the solutions, Humes et al. (2009) proposed that the auditory training can be considered in aural rehabilitation for hearing aid users for improving listening capabilities in noisy conditions.

To date, not many studies have performed auditory training for improving spatial hearing abilities. A comprehensive understanding of hearing loss is crucial for designing an intervention program (Bahng \& Lee, 2020). Firszt et al. (2015) developed the localizationspecific auditory training for unilateral severe to profound hearing loss and reported that eight out of 11 participants' spatial hearing abilities improved significantly. The auditory training was composed of five training sessions, and localization cues decreased 
gradually across sessions. Nawaz et al. (2014) also reported improved localization accuracy following 3 months of auditory training in one participant wearing a single cochlear implant (CI). For the auditory training, the participant listened to the speech in noise sentence using a direct audio input cable to the $\mathrm{CI}$ and one earphone.

To our knowledge, no study has been reported the auditory training for spatial hearing ability in bilateral sensorineural hearing loss. In the present study, we conducted a pilot study of auditory training for improving spatial ability in two elderly hearingimpaired listeners wearing hearing aids. The auditory training consisted of 16 sessions where, in each training session, the target

Table 1. Pure-tone average thresholds of two participants

\begin{tabular}{lcccccc}
\hline & \multicolumn{6}{c}{ Mean thresholds (dB HL) in frequency $(\mathrm{kHz})$} \\
\cline { 2 - 7 } & 0.25 & 0.5 & 1 & 2 & 4 & 6 \\
\hline Subject 1 & & & & & & \\
R & 35 & 40 & 35 & 55 & 55 & 50 \\
L & 25 & 30 & 35 & 60 & 60 & 60 \\
Subject 2 & & & & & & \\
R & 35 & 40 & 60 & 70 & 75 & 65 \\
L & 40 & 45 & 60 & 70 & 75 & 80 \\
\hline
\end{tabular}

R: right ear, L: left ear speech was presented from $0^{\circ}$ azimuth in a quiet environment. The directions of competing speech/noise changed between $0^{\circ}$ to $\pm 90^{\circ}$ azimuth angles. This study also examined if auditory training could improve the spatial hearing ability of the participants. Training efficacy was tested by examining objective changes in pre-training versus post-training measures of spatial processing skills, such as the speech reception threshold (SRT) and speech release from masking (SRM) of the two participants.

\section{CASE REPORT}

\section{Participants}

Two hearing-impaired listeners participated in a total of 16 training sessions. Each training session was composed of 40 minutes, twice a week. Table 1 shows pure-tone average thresholds across six frequencies $(0.25,0.5,1,2,4$, and $6 \mathrm{kHz})$ for the left and right ears for each participant. Also, Table 2 shows the information about age, duration of hearing aid use, type, and brand of hearing aids.

\section{Training procedures}

Auditory training for spatial hearing loss was conducted in a quiet room using the two portable bluetooth speakers (as illustrated in Figure 1). The auditory training consisted of 16 sessions where

Table 2. Age, duration of hearing aid use, type and brand of hearing aids in two participants

\begin{tabular}{lcclcc}
\hline & Age $(\mathrm{yr})$ & Duration of hearing aid use $(\mathrm{yr})$ & \multicolumn{1}{c}{ Type of hearing aids } & Brand of hearing aids \\
\hline Subject 1 & 78 & 3 & Receiver in the ear with custom earmolds & Unitron \\
Subject 2 & 75 & 1 & CIC type & Oticon \\
\hline
\end{tabular}

CIC: completely in the canal

(Part 4) Competing noise at $90^{\circ}$ azimuth
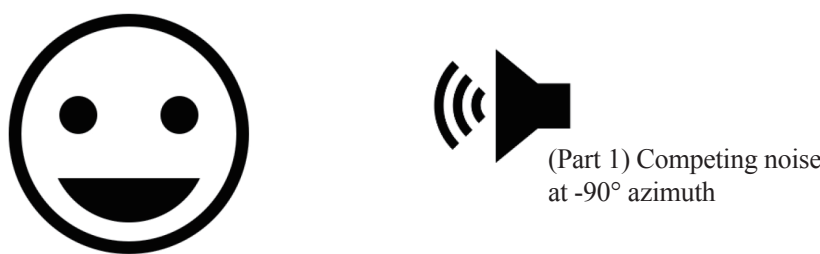

(Part 5) Competing noise at $45^{\circ}$ azimuth
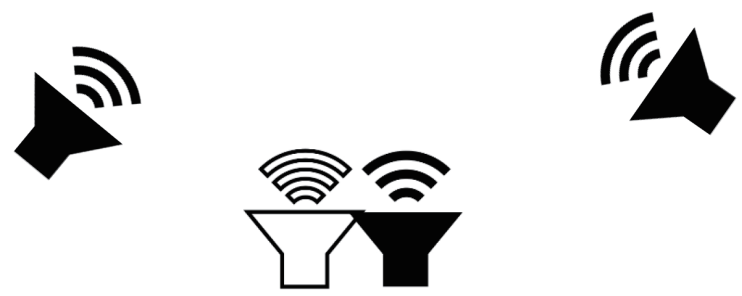

(Part 2) Competing noise at $-45^{\circ}$ azimuth

Target sentences at $0^{\circ}$ azimuth

(Part $3 \&$ 6) Competing noise co-located at $0^{\circ}$ azimuth

Figure 1. Test environment for auditory training of spatial hearing ability. 
the degree of difficulty of the training was varied adaptively and systematically in the following ways. First, the training session progressed from enhanced target sentences with better signal-tonoise ratios (SNRs) to degraded target sentences with poor SNRs in an adaptive fashion. While the level of the target speech was held constant, the noise level was adjusted. Secondly, the degree of difficulty of the training was made harder by decreasing the sensation level of target sentences. Finally, the spatial separation between the target and noise masker was reduced from $\pm 90^{\circ}$ through $\pm 45^{\circ}$ to $0^{\circ}$, making the target stream segregation harder. The target signals were presented from $0^{\circ}$ azimuth while the directions of competing speech/noise changed between $0^{\circ}$ to $90^{\circ}$ azimuth angles $\left(0^{\circ}, \pm 45^{\circ}\right.$, and $\left.\pm 90^{\circ}\right)$. In each training session, feedback about the accuracy of the response was supplied every time.

Figure 1 shows an example of two portable bluetooth speakers used in the auditory training for spatial hearing loss. The training session started with presenting the noise masker to the ear with poorer hearing sensitivity (e.g., from $-90^{\circ}$ azimuth $\left.\left[\mathrm{S}_{0} \mathrm{~N}_{-90}\right]\right)$. In the subsequent parts 2 and 3 , competing speech/noise was presented from $-45^{\circ}$ azimuth (e.g., $\mathrm{S}_{0} \mathrm{~N}_{-45}$ ) and then from $0^{\circ}$ (e.g., $\mathrm{S}_{0} \mathrm{~N}_{0}$ ) azimuth angle, making the target stream segregation harder in part 3 than it was in part 2. In part 4 , the noise masker was presented to the ear with better hearing sensitivity (from $90^{\circ}$ azimuth [e.g., $\mathrm{S}_{0} \mathrm{~N}_{+90}$ ]). One thing to bear in mind in parts 4 and 5, was that the SRM could become negative when the noise masker was presented to the better ear (Corbin et al, 2017). It is because the

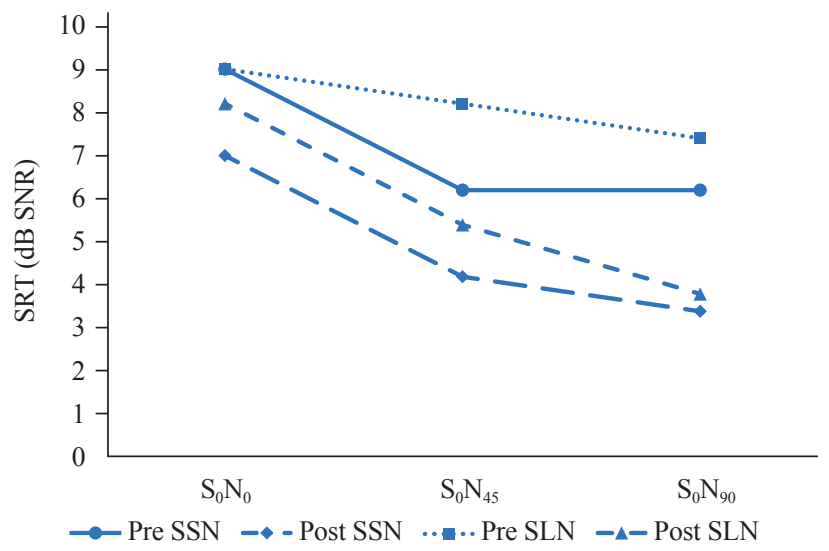

Figure 2. Pre-training versus post-training SRTs for subject 1. SRT: speech reception treshold, SNR: signal-to-noise ratio, $\mathrm{S}_{0} \mathrm{~N}_{0}$ : $\mathrm{S}_{0}$ represents a target speech at $0^{\circ}$ azimuth and $\mathrm{N}_{0}$ represents a noise masker at $0^{\circ}$ azimuth, $\mathrm{S}_{0} \mathrm{~N}_{45}$ : target speech at $0^{\circ}$ azimuth and a noise masker at $45^{\circ}$ azimuth, $\mathrm{S}_{0} \mathrm{~N}_{90}$ : target speech at $0^{\circ}$ azimuth and a noise masker at $90^{\circ}$ azimuth, Pre: pre-trainning, SSN: speech-like noise, Post: post-training, SLN: speech-like noise. participant's spatial hearing can be affected by the noise masker presented to the good ear more than the poor ear. As a result, the participants may need larger $\mathrm{dB}$ SNR in the $\mathrm{S}_{0} \mathrm{~N}_{+45 /+90}$ setup than the $\mathrm{S}_{0} \mathrm{~N}_{0}$ setup to understand correctly $50 \%$ of the target speech in competing speech/noise. In this study, both test subjects had symmetric sensorineural hearing loss, and they did not have a better ear. The auditory training protocol of 16 sessions is presented in Appendix.

\section{Measurements of spatial hearing ability}

Spatial hearing abilities of participants were evaluated in a quiet room using the two portable bluetooth speakers (arranged in the semi-circular horizontal plane at $45^{\circ}$ steps, spinning a full $180^{\circ}$ ). The speakers were placed along with the semi-circular shape, with participants sitting at its centre. Participants were seated in a chair facing the centre of the speaker array at the front $\left(0^{\circ}\right.$ azimuth). These speakers were controlled by a laptop computer via the software package, called Adobe Audition CS6 (Adobe, San Jose, CA, USA), to handle different sound source files.

Each subject's spatial hearing ability was measured by obtaining the SRT and SRM. The SRT is defined in terms of $\mathrm{dB}$ SNR as the relative level of a target sentence, at which $50 \%$ of the words are understood correctly in competing speech/noise. The SRM corresponds to the benefit of target/masker spatial separation. For the test material, the Korean Matrix Tests were used (Kim \& Lee, 2018).

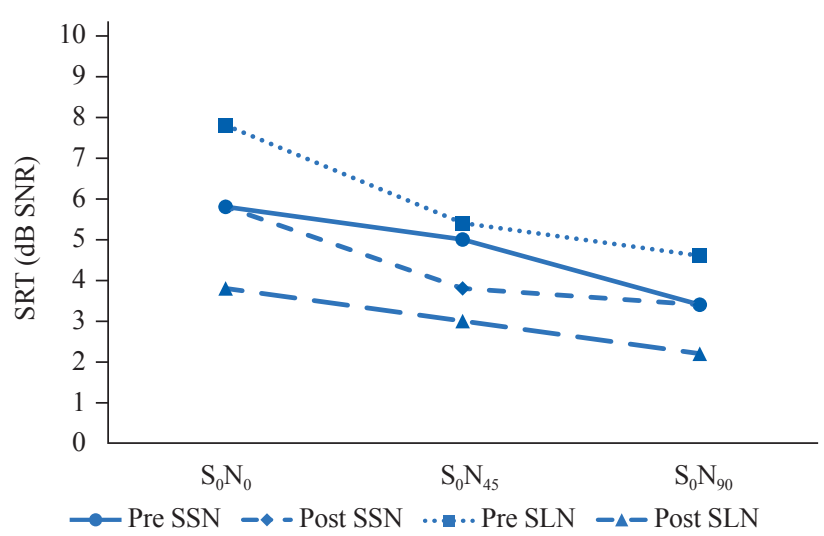

Figure 3. Pre-training versus post-training SRTs for subject 2. SRT: speech reception treshold, $\mathrm{S}_{0} \mathrm{~N}_{0}: \mathrm{S}_{0}$ represents a target speech at $0^{\circ}$ azimuth and $\mathrm{N}_{0}$ represents a noise masker at $0^{\circ}$ azimuth, $\mathrm{S}_{0} \mathrm{~N}_{45}$ : target speech at $0^{\circ}$ azimuth and a noise masker at $45^{\circ}$ azimuth, $\mathrm{S}_{0} \mathrm{~N}_{90}$ : target speech at $0^{\circ}$ azimuth and a noise masker at $90^{\circ}$ azimuth, Pre: pre-training, SSN: speech-like noise, Post: posttraining, SLN: speech-like noise. 


$$
\mathrm{SRM}=\mathrm{SRT}\left[\mathrm{S}_{0} \mathrm{~N}_{0}\right]-\mathrm{SRT}\left[\mathrm{S}_{0} \mathrm{~N}_{ \pm 45 / 90}\right]
$$

where $\mathrm{S}_{0}$ represents a target speech at $0^{\circ}$ azimuth; $\mathrm{N}_{ \pm 45}$ a noise masker at $\pm 45^{\circ}$ azimuth; $\mathrm{N}_{ \pm 90}$ a noise masker at $\pm 90^{\circ}$ azimuth, respectively.

SRT and SRM were measured using two different types of noise: the speech-like noise (SLN) and steady-state noise (SSN). SSN masker used in this paper was speech-shaped stationary noise with a long-term spectrum that matched the long-term spectrum of the target speech material derived from an overlay of 300 sentences of the Korean matrix sentences. The SSN had a higher gain level of the more intense low frequencies than the weaker high frequencies. The steady-state speech-shaped noise does not reflect the dynamic features of real speech signals. However, the speech-shaped noise is generally regarded as a better choice than white noise, because it has a better representation of the gain for normal speech.

SLN masker used in this paper was the two-talker speech masker that consisted of two different edited recordings of voices of two talkers (one female and one male) on various topics. Here, 1) each record was edited to remove short temporal gaps of 200 milliseconds or greater between the words; and then 2) two recordings were mixed in an overlapping manner to counteract the glimpsing effect. Speech stimuli and noise masker had a resolution of 32 bits and a sampling rate of $44.1 \mathrm{kHz}$. These files were rootmean-square normalized to have multiple tracks sound equally loud. The volume was adjusted so that the loudest peak of speech stimuli was equal to the maximum signal used in the noise masker.

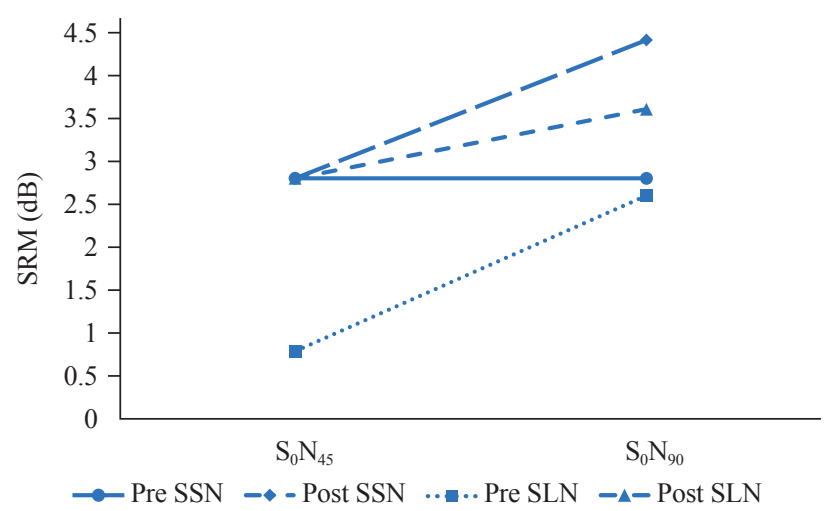

Figure 4. Pre-training versus post-training SRMs for subject 1. SRM: spatial release from masking, $\mathrm{S}_{0} \mathrm{~N}_{45}$ : target speech at $0^{\circ}$ azimuth and a noise masker at $45^{\circ}$ azimuth, $\mathrm{S}_{0} \mathrm{~N}_{90}$ : target speech at $0^{\circ}$ azimuth and a noise masker at $90^{\circ}$ azimuth, Pre: pre-training, SSN: speech-like noise, Post: post-training, SLN: speech-like noise.

\section{Outcomes of the auditory training for spatial hearing loss}

The efficacy of the auditory training was tested by examining the pre-training versus post-training measures of spatial hearing skills, such as SRT and SRM, of the two participants. We did not statistically analyse the data due to a small number of subjects. The comparison of pre-training versus post-training SRTs in Figure 2 \& 3 showed that the participants' speech perception abilities have improved at all conditions regardless of types of noise. Figure $4 \& 5$ indicated the pre-training versus post-training SRMs. Even though two subjects' speech perception abilities increased, only the SRMs of subject 1 were improved.

Subject 1 performed considerably better after the training in every aspect in that both SRT and SRM improved at each spatial location in all noise conditions than before the training where the pre-training SRT of subject 1 was at the bottom of the test range. Notably, SRT of subject 1 improved $3.6 \mathrm{~dB}$ at the condition of using SLN at $\mathrm{S}_{0} \mathrm{~N}_{90}$. In the subject 1 case, SRM improvement was more prominent in SLN. In the subject 2 case, SRT scores improved in all noise conditions. However, SRM has not changed after the training. The results of subject 2 revealed that improvement in SRT does not always coincide with improved SRM.

\section{DISCUSSIONS}

It is known that the ability to localize sounds, which is one of the basic building blocks of spatial hearing, tends to diminish when binaural hearing mechanisms are disrupted (Moossavi et al., 2017). Thus, Firszt et al. (2015) suggested the inclusion of sound localization training in the rehabilitation plan for people with

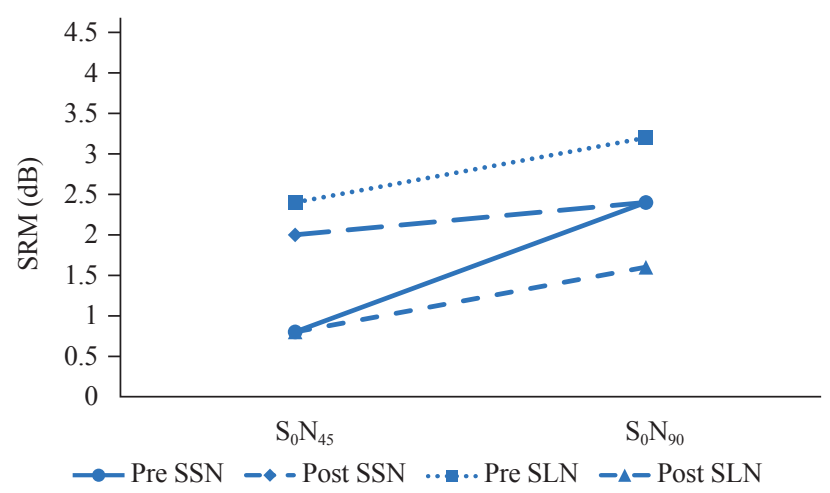

Figure 5. Pre-training versus post-training SRMs for subject 2. SRM: spatial release from masking, $\mathrm{S}_{0} \mathrm{~N}_{45}$ : target speech at $0^{\circ}$ azimuth and a noise masker at $45^{\circ}$ azimuth, $\mathrm{S}_{0} \mathrm{~N}_{90}$ : target speech at $0^{\circ}$ azimuth and a noise masker at $90^{\circ}$ azimuth, Pre: pre-training, SSN: speech-like noise, Post: post-training, SLN: speech-like noise. 
unilateral severe-to-profound hearing loss.

In this study, we examined whether the auditory training directed explicitly at improving spatial hearing skills could improve the spatial hearing abilities of the hearing-impaired participants. The comparison of pre-training versus post-training measures of SRM of the subject 1 revealed that the participant's spatial hearing skills had improved 2.8 to $3.6 \mathrm{~dB}$ in SSN and 1.6 to $4.4 \mathrm{~dB}$ in the SLN at the condition of $\mathrm{S}_{0} \mathrm{~N}_{90}$, respectively. The results of subject 2 , on the other hand, showed the improvement in SRT after the training, but SRM did not change. In which case, spatial hearing skills did not improve evidently.

The ability of hearing-impaired listeners to use interaural cues to segregate the target speech stream from distracting noises can improve with the addition of higher frequency information (Levy et al., 2015). The presence of high-frequency hearing in the participants can be an essential factor to consider when conducting the auditory training of spatial hearing loss. Since the head-shadow effect (equivalent to IID) is the largest at the high-frequency region, it may affect the effectiveness of auditory strategies directed at specifically improving spatial hearing skills. Before the auditory training for spatial hearing ability, we tried to compensate for high-frequency hearing loss by hearing aid technology for both participants. As shown in Table 1, subject 1 (aged 78 years) had moderate-tomoderately severe high-frequency hearing loss, whereas subject 2 (aged 75 years) had moderate high-frequency hearing loss. To compensate for the high-frequency hearing loss, features of hearing aid technologies, such as frequency compression or composition, were used to provide the participants with the additional highfrequency information. Subject 1 with the receiver-in-the-canaltype hearing aids (HAs) was fitted with custom earmolds to reduce the chances of acoustic feedback to ensure the availability of higher frequency information as well as the low-frequency inter-aural timing cues. Subject 2 was fitted with completely in the canal (CIC)-type HAs with frequency compression turned on to provide enough high-frequency amplification. The HAs had frequency composition tuned on to help the participants process high-frequency speech cues effectively. We should have assessed the real ear aided responses for checking high frequency sounds compensation, but we did not. It is the limitation of this study.

It is generally accepted that speech perception in the SSN is traditionally associated with the energetic masking (EM). At the same time, that of the SLN typically involves both EM and informational masking (IM) (Lidestam et al., 2014). In the present case, it was found that different noise types have significantly different effects on SRT. However, the changes in SRM induced by the different masking effects of different noise types were negligible in both participants. This might be because both subjects did not take the full advantages of target/masker spatial separation in complex listening environments, due to the masking effects of noise and hearing loss.

Although we could not reach a solid conclusion on the effectiveness of the auditory training for spatial hearing loss due to a small sample size limitation, we found the significance of this study in ensuring that the auditory training could lead to an increase in the perception of speech under complex listening environments. Both participants reported that the overall experience of the auditory training had boosted self-confidence in complex listening situations and the satisfactory level of hearing aid performance. This kind of self-report about the training outcomes can be an equally important measure of the training efficacy as objective measures. The results of this paper suggested the inclusion of the auditory training for spatial hearing loss in the rehabilitation plan for people with hearing loss.

\section{Ethical Statement}

This study was approved by the Institutional Review Board of Hallym University of Graduate Studies (HUGSAUD \#265830).

\section{Acknowledgments}

Authors thank to all participants.

\section{Declaration of Conflicting Interests}

Junghwa Bahng, a contributing editor of the Audiology and Speech Research, was not involved in the editorial evaluation or decision to publish this article. All remaining authors have declared no conflicts of interest.

\section{Funding}

This work was supported by the Ministry of Education of the Republic of Korea and the National Research Foundation of Korea (NRF-2019S1A5A2A01039904).

\section{Author Contributions}

Conceptualization: Joon Shin, Junghwa Bahng. Data collection: Junghwa Bahng. Formal analysis: Joon Shin, Junghwa Bahng. Funding acquisition: Junghwa Bahng. 
Methodology: Joon Shin. Writing-original draft: Joon Shin.

Writing-review \& editing: Junghwa Bahng. Approval of final manuscript: Junghwa Bahng.

\section{ORCIDiD}

$\begin{array}{ll}\text { Joon Shin } & \text { https://orcid.org/0000-0002-9012-6357 } \\ \text { Junghwa Bahng } & \text { https://orcid.org/0000-0002-5265-3586 }\end{array}$

\section{REFERENCES}

Ahlstrom, J. B., Horwitz, A. R., \& Dubno, J. R. (2009). Spatial benefit of bilateral hearing aids. Ear and Hearing, 30(2), 203-218.

Akeroyd, M. A. \& Whitmer, W. M. (2011). Spatial hearing and hearing aids. ENT and Audiology News, 20(5), 76-79.

Bahng, J. \& Lee, C. H. (2020). Topic modeling for analyzing patients' perceptions and concerns of hearing loss on social Q\&A sites: Incorporating patients' perspective. International Journal of Environmental Research and Public Health, 17(17), 6209.

Corbin, N. E., Buss, E., \& Leibold, L. J. (2017). Spatial release from masking in children: Effects of simulated unilateral hearing loss. Ear and Hearing, 38(2), 223-235.

Chang, S. \& Lee, J. (2016). Development of auditory training tool for adults using sentences. Audiology and Speech Research, 12(2), 8996.

Firszt, J. B., Reeder, R. M., Dwyer, N. Y., Burton, H., \& Holden, L. K. (2015). Localization training results in individuals with unilateral severe to profound hearing loss. Hearing Research, 319, 48-55.

Glyde, H., Cameron, S., Dillon, H., Hickson, L., \& Seeto, M. (2013). The effects of hearing impairment and aging on spatial processing. Ear and Hearing, 34(1), 15-28.

Humes, L. E., Burk, M. H., Strauser, L. E., \& Kinney, D. L. (2009). Development and efficacy of a frequent-word auditory training protocol for older adults with impaired hearing. Ear and Hearing, 30(5), 613-627.

Kim, K. H. \& Lee, J. H. (2018). Evaluation of the Korean matrix sentence test: Verification of the list equivalence and the effect of word position. Audiology and Speech Research, 14(2), 100-107.

Kim, N. K. \& Bahng, J. (2017). Development of a story based auditory training tool and evaluation of the training efficacy for adult hearing impaired listeners. Audiology and Speech Research, 13(2), 133-140.

Koehnke, J. \& Besing, J. M. (2001). The effects of aging on binaural and spatial hearing. Seminars in Hearing, 22(3), 241-254.

Levy, S. C., Freed, D. J., Nilsson, M., Moore, B. C., \& Puria, S. (2015). Extended high-frequency bandwidth improves speech reception in the presence of spatially separated masking speech. Ear and Hearing, 36(5), e214-e224.

Lidestam, B., Holgersson, J., \& Moradi, S. (2014). Comparison of informational vs. energetic masking effects on speechreading performance. Frontiers in Psychology, 5, 639.

Moossavi, A., Abdollahi, F. Z., \& Lotfi, Y. (2017). Spatial auditory processing in children with central auditory processing disorders. Auditory and Vestibular Research, 26(2), 56-63.

Nawaz, S., McNeill, C., \& Greenberg, S. L. (2014). Improving sound localization after cochlear implantation and auditory training for the management of single-sided deafness. Otology \& Neurotology, 35(2), 271-276.

Neher, T., Behrens, T., Carlile, S., Jin, C., Kragelund, L., Petersen, A. S., et al. (2009). Benefit from spatial separation of multiple talkers in bilateral hearing-aid users: Effects of hearing loss, age, and cognition. International Journal of Audiology, 48(11), 758-774. 


\section{APPENDIX}

The auditory training protocol

\begin{tabular}{|c|c|c|c|}
\hline Session & Loudspeaker azimuth $\left(\mathrm{S}_{0} \mathrm{~N}_{\text {degree }}\right)$ & SNR conditions & Auditory training materials \\
\hline 1 & $\mathrm{~S}_{0} \mathrm{~N}_{90}$ & Quiet \& $10 \mathrm{~dB}$ SNR & 20 sentences \& 2 stories \\
\hline 2 & $\mathrm{~S}_{0} \mathrm{~N}_{-90}$ & Quiet \& $10 \mathrm{~dB}$ SNR & 20 sentences $\& 2$ stories \\
\hline 3 & $\mathrm{~S}_{0} \mathrm{~N}_{45}$ & Quiet \& $10 \mathrm{~dB}$ SNR & 20 sentences \& 2 stories \\
\hline 4 & $\mathrm{~S}_{0} \mathrm{~N}_{-45}$ & Quiet \& $10 \mathrm{~dB}$ SNR & 20 sentences $\& 2$ stories \\
\hline 5 & $\mathrm{~S}_{0} \mathrm{~N}_{0}$ & Quiet \& $10 \mathrm{~dB}$ SNR & 20 sentences $\& 2$ stories \\
\hline 6 & $\mathrm{~S}_{0} \mathrm{~N}_{90}$ & $10 \mathrm{~dB}$ SNR \& 5 dB SNR & 15 sentences $\& 2$ stories \\
\hline 7 & $\mathrm{~S}_{0} \mathrm{~N}_{-90}$ & 10dB SNR \& 5 dB SNR & 15 sentences $\& 2$ stories \\
\hline 8 & $\mathrm{~S}_{0} \mathrm{~N}_{45}$ & $10 \mathrm{~dB}$ SNR \& $5 \mathrm{~dB}$ SNR & 15 sentences $\& 2$ stories \\
\hline 9 & $\mathrm{~S}_{0} \mathrm{~N}_{-45}$ & $10 \mathrm{~dB}$ SNR \& $5 \mathrm{~dB}$ SNR & 15 sentences \& 2 stories \\
\hline 10 & $\mathrm{~S}_{0} \mathrm{~N}_{0}$ & $10 \mathrm{~dB}$ SNR \& $5 \mathrm{~dB}$ SNR & 15 sentences $\& 2$ stories \\
\hline 11 & $\mathrm{~S}_{0} \mathrm{~N}_{90}$ & $5 \mathrm{~dB}$ SNR \& $0 \mathrm{~dB}$ SNR & 10 sentences \& 1 story \\
\hline 12 & $\mathrm{~S}_{0} \mathrm{~N}_{-90}$ & $5 \mathrm{~dB}$ SNR \& 0 dB SNR & 10 sentences \& 1 story \\
\hline 13 & $\mathrm{~S}_{0} \mathrm{~N}_{45}$ & $5 \mathrm{~dB}$ SNR \& 0 dB SNR & 10 sentences \&1 story \\
\hline 14 & $\mathrm{~S}_{0} \mathrm{~N}_{-45}$ & $5 \mathrm{~dB}$ SNR \& 0 dB SNR & 10 sentences \& 1 story \\
\hline 15 & $\mathrm{~S}_{0} \mathrm{~N}_{0}$ & $5 \mathrm{~dB}$ SNR \& 0 dB SNR & 10 sentences \& 1 story \\
\hline 16 & Random & Random & 20 sentences $\& 2$ storeis \\
\hline
\end{tabular}

Signal (sentences \& stories) always presented at 0 azimuths. The training session started with presenting the noise masker to the ear with poorer hearing sensitivity. If participants performed under $60 \%$ performance at a condition, the clinician changed to easier conditions and conducted the auditory training. For example, at $\mathrm{S}_{0} \mathrm{~N}_{90}$ with $0 \mathrm{~dB}$ SNR, if the participant showed $50 \%$ performance, the condition was changed to $\mathrm{S}_{0} \mathrm{~N}_{90}$ with $5 \mathrm{~dB}$ SNR. Recorded sentences and stories were used (Chang \& Lee, 2016; Kim \& Bahng, 2017). The auditory training was conducted twice a week, and each session was 40 minutes. SNR: signal to noise ratio 